\title{
Conventional Synthetic Dmards in Psoriatic Arthritis: Changing Practice in Biologic Era; Real-Life Results from HURBI0-PsA Registry
}

Emre Bilgin, [MD]

ORCID: 0000-0002-2260-4660

Umut Kalyoncu, [MD]

ORCID: 0000-0001-7129-2109

${ }^{1}$ Hacettepe University, Department of Internal Medicine, Division of Rheumatology, Ankara, Turkey.

\section{Corresponding Author: Emre Bilgin}

Hacettepe University Faculty of Medicine, Department of Internal Medicine, Division of Rheumatology, Turkey. E-mail: dr.emrebilgin@gmail.com

https://doi.org/10.32552/2021.ActaMedica.623

\section{n ABSTRACT Con}

Objectives: Psoriatic arthritis is a chronic musculoskeletal disorder which may affect skin, joints, bone and enthesis. Conventional synthetic disease modifying anti-rheumatic drugs are first-line treatment options and biologic disease modifying anti-rheumatic drugs are recommended in psoriatic arthritis patients who are intolerant/not controlled well with conventional synthetic disease modifying anti-rheumatic drugs. Although survival data of the conventional synthetic disease modifying anti-rheumatic drugs without concomitant biologic disease modifying anti-rheumatic drugs are available, the effect of biologic disease modifying anti-rheumatic drugs on the retention of conventional synthetic disease modifying anti-rheumatic drugs is still a question of interest.

Materials and Methods: Psoriatic arthritis patients who received at least 1 dose of biologic disease modifying anti-rheumatic drugs, using at least 1 conventional synthetic disease modifying anti-rheumatic drugs (methotrexate, leflunomide, hydroxychloroquine and sulfasalazine) at the time of biologic disease modifying anti-rheumatic drugs starting visit and registered in the Hacettepe University BIOlogical DatabasePsoriatic Arthritis were included in this retrospective longitudinal analysis. Demographic and disease-specific data at first and last followup visit were collected. Unadjusted retention rate of each conventional synthetic disease modifying anti-rheumatic drugs was assessed. Overall prescription of conventional synthetic disease modifying anti-rheumatic drugs at first and last follow-up visit were compared.

Results: A total of 266 (191(71.8\%) female) patients was included. Median follow-up duration under biologic treatment was 43.4 (19.480.1) months. Median retention duration of each conventional synthetic disease modifying anti-rheumatic drugs were similar. Between the first and last visit; there was a $29.3 \%$ decrease in methotrexate use $161.7 \%$ vs. $43.6 \% ; p<0.001), 8.4 \%$ decrease in leflunomide use $(31.2 \%$ vs. $28.6 \%$; $p=0.30), 30.0 \%$ decrease in sulfasalazine use $(11.3 \%$ vs. $7.9 \% ; p=0.05)$, $31.1 \%$ decrease in hydroxychloroquine use ( $16.9 \%$ vs. $11.7 \% ; p=0.001)$, $12.5 \%$ decrease in glucocorticoids use $(51.1 \%$ vs. $44.7 \% ; p=0.015)$. At last visit, 59 (22.2\%) patients were conventional synthetic disease modifying anti-rheumatic drugs -free: 20 (7.5\%) patients were using only glucocorticoids, 39 (14.7\%) patients were conventional synthetic disease modifying anti-rheumatic drugs + glucocorticoid-free.

Conclusion: Although conventional synthetic disease modifying antirheumatic drugs were significantly discontinued in an important percent of patients after the initiation of biologic disease modifying anti-rheumatic drugs, percentage of patients using glucocorticoids at last visit was still high. Studies aiming to demonstrate when, in whom and how to discontinue conventional synthetic disease modifying antirheumatic drugs are needed.

Keywords: Psoriatic arthritis, antirheumatic agents, follow-up studies 


\section{INTRODUCTION}

Psoriatic arthritis (PsA), is a multisystemic, chronic, inflammatory musculoskeletal disorder which is associated with psoriasis (PsO). In addition to articular involvement; nail involvement, enthesitis and dactylitis may be added to clinical picture [1].

By most of the international organization guidelines, conventional synthetic disease modifying antirheumatic drugs (csDMARDs) are referred as the first-line treatment options in the management of PsA $[2,3]$. These csDMARDs are methotrexate (MTX), leflunomide (LEF) and sulfasalazine (SSZ). Although hydroxychloroquine $(\mathrm{HQ})$ is not take its place in the recommendations due to an earlier study which has suggested a risk of exacerbation in $\mathrm{PsO}$, we consider $\mathrm{HQ}$ as a favorable treatment option [4]. Although these drugs showed borderline efficacy in randomized clinical trials, their long retention duration and rates in real-life studies contradict [5].

Biologic DMARDs (anti-tumor necrosis factor, antiinterleukin 17, anti-interleukin 12/23) are real gamechangers in the management of PsA for the last two decades. They are recommended in PsA patients who are not adequately responsive/unresponsive or intolerant to csDMARDs [2, 3]. Although current data suggest no additional benefit of csDMARDs (especially MTX) when combined with bDMARDs, they are generally continued with bDMARDs at least during the initial period of bDMARD treatment [6]. However, data on the effect of bDMARDs on the retention of csDMARDs is scarce.

The aim of this study was to assess the overall retention of csDMARDs which was prescribed at the bDMARD starting visit and define the change in the prescription patterns of csDMARDs between the bDMARD starting visit and last control visit.

\section{MATERIALS and METHODS}

We conducted this retrospective longitudinal analysis with PsA patients who received at least 1 dose of biologic DMARDs, using at least 1 csDMARD (methotrexate, leflunomide, hydroxychloroquine, sulfasalazine) at the time of bDMARDs starting visit and registered in the Hacettepe University BIOlogical Database-PsA (HURBIO-PsA) which was settled in 2005. Diagnosis of PsA was established by treating physician with taking into account the history, physical examination, laboratory and imaging of the patients.

Of 524 patients registered to HURBIO-PSA, 20 of them had incomplete first visit data and 88 did not have control visit data. 416 of them had compete data. Of these 416 patients, 150 patients were not under csDMARDs (98 patients had no csDMARDs including glucocorticoids (GCs), 52 patients had only GCs without csDMARDs). Remaining 266 patients were included in the final analysis.

\section{Data Collection}

\section{Demographic Data and Population Characteristics}

Following demographic datawerecollected:gender, age, $\mathrm{PsO}$ and PsA disease duration, family history of psoriatic disease (PsO and/or PsA), educational and marital status, smoking history, body mass index $(\mathrm{BMI})$, the frequency of hypertension, coronary artery disease and diabetes mellitus. Distribution of the first prescribed bDMARDs and disease activity parameters [erythrocyte sedimentation rate (ESR) $(\mathrm{mm} / \mathrm{h}), \mathrm{C}$-reactive protein $(\mathrm{CRP})(\mathrm{mg} / \mathrm{dl})$, disease activity score (DAS) 28-ESR, health assessment questionnaire-disability index (HAQ-DI), Bath Ankylosing spondylitis Disease Activity Index (BASDAI), Bath Ankylosing spondylitis Functional Index (BASFI), tender and swollen joint count (28 joints), patient global-visual analogue scale assessment (VAS) $(0-100 \mathrm{~mm})$ and Psoriatic arthritis impact of disease (PSAID)] at the first visit and last follow-up visit were also assessed.

\section{Assessment of Retention of csDMARDs}

Methotrexate, leflunomide, hydroxychloroquine and sulfasalazine were the csDMARDs included in the analysis. Unadjusted retention rate of the each of these csDMARDs was assessed. Besides, we compared the overall prescription of csDMARDs (both for each csDMARDs and whether they were prescribed alone $[ \pm G C s]$ or in combination). For this overall comparison, percentage of patients who was prescribed the each csDMARDs at first visit was compared with the percentage of patients who was prescribed that csDMARD at last visit.

Our study is compliant with the Helsinki Declaration and approved by Hacettepe University ethical committee (GO 21/103. Date: 14/01/2021). 


\section{Statistical Analyses}

Statistical analysis was performed using the Statistical Package for the Social Sciences software (version 25.0; IBM Corporation, Armonk, NY, USA). The variables were investigated using visual (histogram, probability plots) and analytic methods (Kolmogorov-Smirnov, skewness and curtosis) to determine whether they are normally distributed or not. The data of descriptive analysis were expressed as either mean \pm standard deviation (SD) or the median, interquartile range (IQR). Categorical variables at first and last visit were compared with the McNemar test. The paired sample Student- $t$ test or Wilcoxon-rank test was used to compare the normal and non-normally distributed continuous at first and last visit, respectively. Retention rate of each csDMARD was determined via Kaplan-Meier curve by assuming the status of csDMARD use at last visit as dependent variable and duration between the first and last visit as time variable.

\section{RESULTS}

\section{Demographic Data and Population Characteristics}

A total of 266 patients was included in the analysis. Median age was 48.5 (40.0-59.0) years [median (interquartile range)] and 191 (71.8\%) of patients were female. Median PsA and PsO disease duration were 9.0 (5.0-13.0) and 18.0 (11.0-25.0) years, respectively. 97 (36.5\%) patients had a family history of psoriatic disease (PsO and/or PsA). 144 (54.1\%) patients were active or ex-smoker. Hypertension, diabetes mellitus and obesity (BMI>30) were prevalent in $25.6 \%, 11.3 \%$, and $44.7 \%$ of patients, respectively. Details were given in Table 1.

Median follow-up duration under biologic treatment was 43.4(19.4-80.1) months. Distribution of first biologic DMARD was as follows: 123 (46.2\%) adalimumab, 56 (21.1\%) etanercept, 47 (17.8\%) infliximab, 25 (9.4\%) golimumab and certolizumab pegol 15 (5.8\%). Baseline and last follow-up disease activity parameters and composite index scores were given in Table 1 and all parameters were significantly decreased at follow-up visit $(p<0.01$ for all parameters).

\section{Retention of csDMARDs}

At first visit, number of patients using each CsDMARDs were as follows: 164 (61.7\%) methotrexate, 83 (31.2\%) leflunomide, 30 (11.3\%) sulfasalazine and $45(16.9 \%)$ hydroxychloroquine. $136(51.1 \%)$ patients had concomitant GCs. While $217(81.6 \%)$ patients had mono-csDMARD \pm GCs, $49(18.4 \%)$ patients had combined-csDMARD \pm GCs. Median retention duration of each csDMARD were similar and about 110 months. Kaplan-Meier curves for the retention of each csDMARD were given in the Figure 1.

During follow-up, methotrexate was discontinued for 54 patients and started to 6 patients, resulting 116 patients to use methotrexate. There was a $29.3 \%$ decrease in methotrexate use at last visit relative to first visit $(61.7 \%$ vs. $43.6 \% ; p<0.001)$. Leflunomide was discontinued for 20 patients and started to 13 patients, resulting 76 patients to use leflunomide. There was an $8.4 \%$ decrease in leflunomide use at last visit relative to first visit (31.2\% vs. $28.6 \% ; p=0.30)$. Sulfasalazine was discontinued for 13 patients and started to 8 patients, resulting 21 patients to use sulfasalazine. There was a $30.0 \%$ decrease in sulfasalazine use at last visit relative to first visit $(11.3 \%$ vs. $7.9 \%$; $p=0.05$ ). Hydroxychloroquine was discontinued for 15 patients and started to 1 patient resulting 31 patients to use hydroxychloroquine. There was a $31.1 \%$ decrease in hydroxychloroquine use at last visit relative to first visit ( $16.9 \%$ vs. $11.7 \%$; $p=0.001)$. There was also a $12.5 \%$ decrease in GCs use at last visit relative to first visit ( $51.1 \%$ vs. $44.7 \% ; p=0.015)$. Overall, mono-csDMARD and combined-csDMARD percentages were significantly decreased at last visit relative to first visit ( $\Delta$ percentage: $22.0 \%$ and $32.7 \%, p<0.001$ and $p=0.001$, respectively). At last visit, 59 (22.2\%) patients were csDMARDfree: $20(7.5 \%)$ patients were using only GCs, 39 (14.7\%) patients were csDMARD + GC free. Details were given in Table 2. There were no clinical or demographic difference between patients who continued or discontinued csDMARDs.

\section{DISCUSSION}

In this retrospective longitudinal analysis, we found concomitant csDMARDs use in patients who are under bDMARDs were significantly tapered after a median follow-up of 43.4 months. About one fourth of the patients were csDMARD-free at last control visit. Crude retention rates of each csDMARDs were similar. 
Table 1. Patient characteristics $(\mathrm{N}=266)$

\begin{tabular}{|c|c|c|c|c|}
\hline Female (n,\%) & \multicolumn{4}{|c|}{$191 / 266(71.8)$} \\
\hline Age, years & \multicolumn{4}{|c|}{$48.5(40.0-59.0)$} \\
\hline PsA disease duration, years & \multicolumn{4}{|c|}{$9.0(5.0-13.0)$} \\
\hline PsO disease duration, years $(n=213)$ & \multicolumn{4}{|c|}{$18.0(11.0-25.0)$} \\
\hline Follow-up duration under biologic treatment, months & \multicolumn{4}{|c|}{$43.4(19.4-80.1)$} \\
\hline Family history of psoriatic disease (n,\%) & \multicolumn{4}{|c|}{$97(36.5)$} \\
\hline \multicolumn{5}{|l|}{ Marital status (n, \%) } \\
\hline Married & \multicolumn{4}{|c|}{$227(85.3)$} \\
\hline Single & \multicolumn{4}{|c|}{$39(14.7)$} \\
\hline \multicolumn{5}{|l|}{ Educational status ( $\mathrm{n}, \%)$} \\
\hline$<$ High school & \multicolumn{4}{|c|}{$113(42.5)$} \\
\hline$\geq$ High school & \multicolumn{4}{|c|}{$153(57.5)$} \\
\hline \multicolumn{5}{|l|}{ Smoking (n, \%) } \\
\hline Active or ex-smoker & \multicolumn{4}{|c|}{$144(54.1)$} \\
\hline Never smoked & \multicolumn{4}{|c|}{$122(45.9)$} \\
\hline \multicolumn{5}{|l|}{ BMI } \\
\hline Median & \multicolumn{4}{|c|}{$29.0(25.9-33.8)$} \\
\hline$\geq 30(n, \%)$ & \multicolumn{4}{|c|}{$119(44.7)$} \\
\hline \multicolumn{5}{|l|}{ Comorbidities } \\
\hline Diabetes & \multicolumn{4}{|c|}{$30(11.3)$} \\
\hline Hypertension & \multicolumn{4}{|c|}{$68(25.6)$} \\
\hline Coronary artery disease & \multicolumn{4}{|c|}{$7(2.6)$} \\
\hline \multicolumn{5}{|l|}{ First biologic agent } \\
\hline Adalimumab & \multicolumn{4}{|c|}{$123(46.2)$} \\
\hline Infliximab & \multicolumn{4}{|c|}{$47(17.8)$} \\
\hline Etanercept & \multicolumn{4}{|c|}{$56(21.1)$} \\
\hline Golimumab & \multicolumn{4}{|c|}{$25(9.4)$} \\
\hline Certolizumab pegol & & $15(5.8$ & & \\
\hline Disease activity parameters & Visit at the & prescription (n) & Last $f$ & w-up visit (n) \\
\hline ESR & 218 & $20.0(10.0-37.0)$ & 263 & $16.0(8.0-27.0)$ \\
\hline CRP & 190 & $1.0(0.5-1.8)$ & 260 & $0.5(0.3-0.9)$ \\
\hline DAS28 & 142 & $4.0(3.0-5.1)$ & 256 & $2.6(1.9-3.6)$ \\
\hline HAQ & 125 & $0.7(0.3-1.0)$ & 253 & $0.4(0.1-0.9)$ \\
\hline BASDAI & 139 & $6.1(4.8-7.4)$ & 260 & $3.5(1.5-5.8)$ \\
\hline BASFI & 132 & $4.0(1.6-6.2)$ & 260 & $2.4(0.9-4.9)$ \\
\hline Swollen joint count & 144 & $1.0(0-4.0)$ & 260 & $0(0-0)$ \\
\hline Tender joint count & 143 & $3.0(0-6.0)$ & 260 & $0(0-2.0)$ \\
\hline VAS global & 176 & $60.0(50.0-80.0)$ & 265 & $40.0(20.0-60.0)$ \\
\hline PSAID & 30 & $6.4(5.2-7.3)$ & 33 & $3.6(1.4-5.6)$ \\
\hline
\end{tabular}

BASDAl: Bath Ankylosing spondylitis Disease Activity Index, BASFI: Bath Ankylosing spondylitis Functional Index, BMI: Body mass index, CRP: C-reactive protein, DAS28: Disease Activity Score 28, ESR: Erythrocyte sedimentation rate, HAQ: Health assessment questionnaire, PsA: psoriatic arthritis; PsO: psoriasis, PSAID: Psoriatic arthritis impact of disease, VAS: Visual analogue scale

Data were given as $\mathrm{n}(\%)$ and median (interquartile range).

$\mathrm{p}<0.01$ for each variable when "first visit" and "last follow-up" values were compared by Wilcoxon-rank test. 

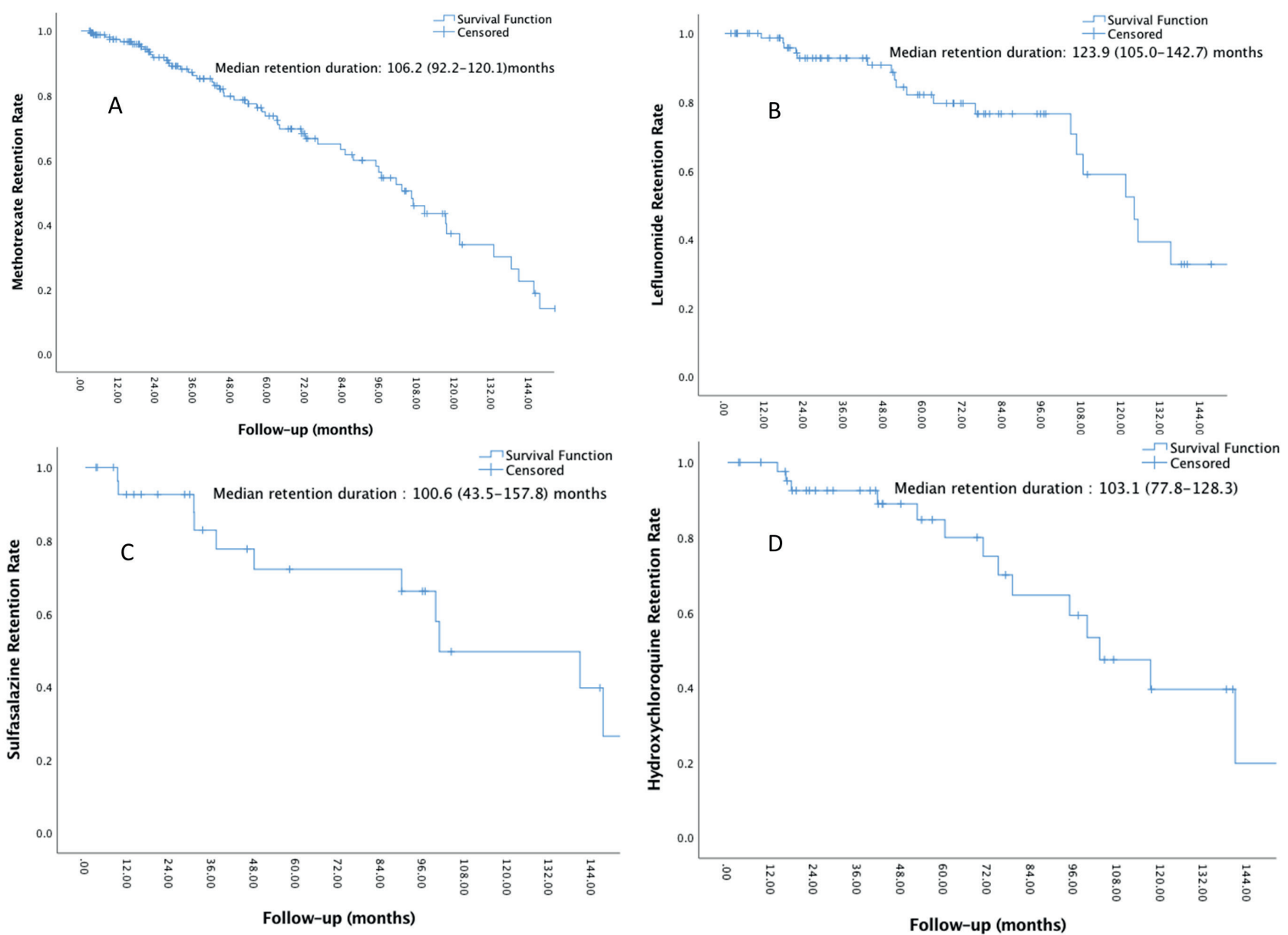

Figure 1. Overall retention of each csDMARD during the follow-up: A) Methotrexate, B) Leflunomide, C) Sulfasalazine, D) Hydroxychloroquine

Table 2. Comparison of the overall prescription of csDMARDs at first and last follow-up visit

\begin{tabular}{|c|c|c|c|c|c|c|c|}
\hline & \multirow{2}{*}{$\begin{array}{c}\text { Duration } \\
\text { between first } \\
\text { and last visit } \\
\text { (median, Q1-Q3) }\end{array}$} & \multirow[t]{2}{*}{ First visit } & \multicolumn{2}{|c|}{$\begin{array}{l}\text { Number of patients } \\
\text { Discontinued/Initiated } \\
\text { drug during follow-up }\end{array}$} & \multirow[t]{2}{*}{ Last visit } & \multirow[t]{2}{*}{$\begin{array}{c}\Delta \text { percentage } \\
(\%)\end{array}$} & \multirow[t]{2}{*}{$\mathrm{p}^{*}$} \\
\hline & & & $\begin{array}{c}\text { Discontinued } \\
\text { (n) }\end{array}$ & Initiated (n) & & & \\
\hline Methotrexate & $40.5(18.6-74.0)$ & $164(61.7)$ & 54 & 6 & $116(43.6)$ & -29.3 & $<0.001$ \\
\hline Leflunomide & $50.9(21.8-20.1)$ & $83(31.2)$ & 20 & 13 & $76(28.6)$ & -8.4 & 0.30 \\
\hline Sulfasalazine & $41.6(15.8-99.6)$ & $30(11.3)$ & 13 & 8 & $21(7.9)$ & -30.0 & 0.05 \\
\hline Hydroxychloroquine & $43.0(21.8-94.7)$ & $45(16.9)$ & 15 & 1 & $31(11.7)$ & -31.1 & 0.001 \\
\hline Glucocorticoids (GCs) & $43.4(19.4-80.1)$ & $136(51.1)$ & 30 & 13 & $119(44.7)$ & -12.5 & 0.015 \\
\hline Mono-csDMARD $\pm \mathrm{GCs}$ & $43.6(19.4-76.7)$ & 217 (81.6) & - & - & $174(65.4)$ & -22.0 & $<0.001$ \\
\hline $\begin{array}{l}\text { Combined-csDMARD } \pm \\
\text { GCs }\end{array}$ & $43.0(20.6-97.1)$ & $49(18.4)$ & - & - & $33(12.4)$ & -32.7 & 0.001 \\
\hline $\begin{array}{l}\text { csDMARD-free (including } \\
\text { GCs) }\end{array}$ & & - & - & - & $39(14.7)$ & - & - \\
\hline Only GCs & & - & - & - & $20(7.5)$ & - & - \\
\hline
\end{tabular}

csDMARD: conventional synthetic Disease Modifying Anti-Rheumatic Drugs, GC: Glucocorticoids ${ }^{*}$ McNemar test (first visit vs. last visit)

$\Delta$ percentage: Change of the overall percentage of the drug prescribed between the last visit and first visit 
In the current perspective and up-to-date treatment guidelines; csDMARDs, especially MTX, stand as the first-line treatmenf option of PsA [2, 3]. Methotrexate is the most commonly prescribed csDMARD in real-life PsA cohorts $[4,5,7]$. Retention of MTX monotherapy in PsA patients has been previously studied in patients who were not under bDMARDs. Although there were contradicting duration of retention, generally the median duration of MTX retention is over 3 years. However; patient characteristics were largely different in these studies compared to our study and in our study, all patients were under bDMARDs- major difference from other studies $[5,8,9]$. Another point regarding MTX is that whether MTX increases the effectiveness of bDMARDs or not. Although latest randomized controlled studies and meta-analysis revealed no additional effect of MTX regarding the bDMARD efficacy, about $40 \%$ of our study cohort was still on MTX[6]. Reasons for this situation need further exploration; however, clinicians' attributes may largely be the cause of this high retention. Leflunomide stands as the second most commonly prescribed csDMARD in our cohort. Efficacy of leflunomide in PsA has been shown in randomized clinical trials and real-life studies $[8,10]$. Some studies found similar drug retention with MTX, which is similar to our study; and in some of the studies, leflunomide had a lower drug retention rate $[8,11]$. In our study, the difference in the rate of leflunomide prescription between the first and last control was not statistically significant. The reason for this was the similar number of patients who stopped and started leflunomide. In case of methotrexate intolerance/ineffectiveness, leflunomide stands as a good option. Sulfasalazine is generally the second most common option is PsA after MTX, however it was least commonly prescribed csDMARD in our cohort [4]. This may be because of the different involvement patterns of our patients, patient characteristics and physician attitudes. Although we found similar retention rate of MTX and SSZ, data suggesting a lower retention rate of SSZ is also recently published [5]. Hydroxychloroquine is not generally recommended and does not take part in the current treatment guidelines. The reason is the historical data which suggests the possible psoriasis-triggering effect of the HQ [12]. However, $\mathrm{HQ}$ is a generally welltolerated and prescribed treatment option in real-life studies. Similarly, it was one of the major csDMARDs in our cohort.

In our cohort, we observed that both monocsDMARD and combined csDMARD regimens were significantly decreased. During follow-up, $22.2 \%$ of the patients become csDMARDs-free who are mainly constitutes from the patients who were using mono-csDMARDs at first visit. This is an important effect of bDMARDs to underlie, because decreasing the number of pill that a chronically ill patient has to take improves the quality life of the patient, also increases the medication compliance. Despite the decrease in the rate of patients under CsDMARDs, there were a high rate of GC use at first and last visit. As we know that metabolic disorders like type 2 diabetes, obesity and hyperlipidemia accompany PsA often [13], clinicians should pay more attention to taper and stop GCs.

There were several limitations to our study. First, there were an inborn risk of biases related to retrospective nature of the study. We could not assess the adverse events related to csDMARDs and causes of discontinuation. Also, we could not assess the effectiveness of csDMARDs separately. Although we provide drug retention data, it was a crude estimation, as we could not know the actual drug cessation dates. Besides, we could not assess the medication compliance of the patients.

In conclusion, there were a significant discontinuation of csDMARDs in a notable percent of patients after starting bDMARDs. However, GC prescription rate was still high. There is still a long way to achieve cSDMARD-free remission and more studies aiming to construct strategies for the cessation of csDMARDs are needed.

\section{CONFLICT Of INTEREST}

Authors declare no conflict of interest. 
[1] Gladman DD, Antoni C, Mease P et al. Psoriatic arthritis: epidemiology, clinical features, course, and outcome. Ann Rheum Dis 2005; 64 (Suppl 2): ii14-17.

[2] Coates LC, Corp N, van der Windt DA et al. GRAPPA Treatment Recommendations: An Update From the 2020 GRAPPA Annual Meeting. J Rheumatol 2021; jrheum.201681.

[3] Gossec L, Baraliakos X, Kerschbaumer A et al. EULAR recommendations for the management of psoriatic arthritis with pharmacological therapies: 2019 update. Ann Rheum Dis 2020; 79(6):700-12.

[4] Kalyoncu U, Bayindir O, Ferhat Oksuz M et al. The Psoriatic Arthritis Registry of Turkey: results of a multicentre registry on 1081 patients. Rheumatology (Oxford) 2017; 56(2):27986.

[5] Jacobs ME, Pouw JN, Welsing $P$ et al. First-line csDMARD monotherapy drug retention in psoriatic arthritis: methotrexate outperforms sulfasalazine. Rheumatology (Oxford) 2021; 60(2):780-84.

[6] XieY, Liu Y, Liu Y. Are biologics combined with methotrexate better than biologics monotherapy in psoriasis and psoriatic arthritis: A meta-analysis of randomized controlled trials. Dermatol Ther 2021:e14926.

[7] Wilsdon TD, Whittle SL, Thynne TR et al.Methotrexate for psoriatic arthritis. Cochrane Database Syst Rev 2019; 1:CD012722.
[8] Landi M, Zaffarana CA, Cerda O et al. Methotrexate and leflunomide survival in patients with psoriatic arthritis. International Journal of Clinical Rheumatology 2018; 13(2):102-07.

[9] Lie E, van der Heijde D, Uhlig T et al. Effectiveness and retention rates of methotrexate in psoriatic arthritis in comparison with methotrexate-treated patients with rheumatoid arthritis. Ann Rheum Dis 2010; 69(4):671-76.

[10] Kaltwasser JP, Nash P, Gladman D et al. Treatment of Psoriatic Arthritis Study G: Efficacy and safety of leflunomide in the treatment of psoriatic arthritis and psoriasis: a multinational, double-blind, randomized, placebo-controlled clinical trial. Arthritis Rheum 2004; 50(6):1939-50.

[11] Malesci $D$, Tirri R, Buono $R$ et al. Leflunomide in psoriatic arthritis: a retrospective study of discontinuation rate in daily clinical practice compared with methotrexate. Clin Exp Rheumatol 2007; 25(6):881-4.

[12] Luzar MJ. Hydroxychloroquine in psoriatic arthropathy: exacerbations of psoriatic skin lesions. J Rheumatol 1982; 9(3):462-4.

[13] Karmacharya P, Ogdie A, Eder L. Psoriatic arthritis and the association with cardiometabolic disease: a narrative review. Ther Adv Musculoskelet Dis 2021; 13:1759720X21998279. 